\title{
Food and nutrient intake across social class in Irish adults
}

\author{
C.M. Donovan ${ }^{1}$, M. Buffini ${ }^{1}$, A.P. Nugent ${ }^{1,2}$, A. Flynn ${ }^{3}$, J. Walton ${ }^{4}$ and B.A. McNulty ${ }^{1}$ \\ ${ }^{1}$ UCD Institute of food and health, University College Dublin, Belfield, Dublin, Ireland, \\ ${ }^{2}$ Institute for Global Food Security, Queens University, Belfast, UK, \\ ${ }^{3}$ School of Food and Nutritional Sciences, University College Cork, Cork, Ireland and \\ ${ }^{4}$ Department Biological Sciences, Munster Technological University, Cork, Ireland
}

Food and nutrient intakes have been shown to follow a socioeconomic gradient whereby higher-quality diets are associated with greater affluence, while nutrient-poor diets are associated with lower socioeconomic status ${ }^{(1)}$. The current Irish Obesity Policy highlights socioeconomic inequalities as a priority ${ }^{(2)}$. The aim of this analysis was to identify differences in dietary intake (excluding supplements) among Irish adults across social classes.

Nationally representative dietary data (4-day, semi-weighed food diary) from n1500 Irish adults, aged 18-90 years who participated in the National Adult Nutrition Survey (NANS; 2008-2010) were analysed. Of the n1500 participants, a sub sample of n1276 provided sufficient socioeconomic information which allowed for classification among one of two groups: higher social class (professional, managerial \& technical or managerial) or lower social class (skilled manual, semi-skilled or unskilled). Statistical analysis was carried out using SPSS v26. Chi-squared and Students t-tests explored differences between social classes in terms of demographics. Multivariate general linear models, adjusted for misreporting of energy and gender, compared food and nutrient intakes of individuals from the two social class groups. The Alternative Healthy Eating Index (AHEI) was applied to assess diet quality.

When classified according to social class, n937 participants met the classification of the higher social class group, while n339 were categorised into the lower social class group. The groups differed significantly $(\mathrm{p}<0.001)$ in terms of gender, with males accounting for $34.1 \%$ and $15.4 \%$, and females accounting for $39.3 \%$ and $11.2 \%$ of the higher and lower social class groups, respectively. Body Mass Index $(B M I)$ also differed significantly $(\mathrm{p}<0.001)$ between the groups with $22 \%$ of the higher and $33 \%$ of the lower social class groups classified as living with obesity $(\mathrm{BMI} \geq 30 \mathrm{~kg} / \mathrm{m} 2)(3)$. Significant differences were identified between the two groups in terms of the percentage energy from total fat ( $\mathrm{p} 0.015)$; saturated fat ( $\mathrm{p} 0.003)$ and mean daily intake of fibre ( $\mathrm{g} / \mathrm{day})(\mathrm{p} 0.009)$. With regards to diet quality, the AHEI identified a significant difference between the two groups, with those from the higher social class group displaying an overall healthier diet score $(p<0.001)$. Food groups which differed significantly between the social class groups, were 'butter, fat spreads and hard cooking fats' and 'chips and processed potatoes', both of which saw higher contributions in the lower social class group.

These findings indicate dietary differences among social class groups in terms of nutrients, food groups and overall diet quality within the Irish population. This provides evidence of the socioeconomic gradient of dietary intake in Ireland. Future research should focus on providing current dietary intakes of the Irish population to assess the social socioeconomic gradient and the impact of policies on such.

\section{Acknowledgements}

The National Adult Nutrition Survey was funded by the Irish Department of Agriculture, Food and the Marine.

\section{References}

1. Darmon N \& Drewnowski A (2008) Am J Clin Nutr, 1107-17

2. Department of Health (2016) published by the stationery office

3. World Health Organisation Body Mass Index Classification [Available at: https://www.euro.who.int/] 